\title{
La percepción social de la violencia y la elaboración de la nota roja en la prensa escrita salvadoreña
}

Lauru García Dueñas

\begin{tabular}{|c|c|}
\hline $\begin{array}{l}\text { Resumen: } \\
\text { La violencia es uno de } \\
\text { Los problemas sociales que más } \\
\text { Los medios de comunicación, en } \\
\text { particular la prensa escrita, relatan } \\
\text { diariamente este tema, tejiendo una } \\
\text { relación de sentido con la percep- } \\
\text { ción social de la violencia. Durante } \\
\text { más de dos años, esta investigadora } \\
\text { estudió el fenómeno partiendo de } \\
\text { un análisis socio-histórico de las } \\
\text { circunstancias que permitieron que } \\
\text { la violencia se convirtiera en una } \\
\text { vorágine, pasando por el análisis de } \\
\text { discurso de diez notas emblemáticas } \\
\text { que marcaron a la opinión pública } \\
\text { entre } 2003 \text { y } 2007 \text { y concluyendo } \\
\text { con un análisis de recepción con tres } \\
\text { grupos de discusión. } \\
\text { Los agentes entrevistados re- } \\
\text { sultaron ser más críticos ante los } \\
\text { medios de comunicación lo que se } \\
\text { podría suponer, sobre todo porque } \\
\text { su 'capital político' les permite tener } \\
\text { una opinión más formada frente a los } \\
\text { problemas de la realidad nacional. } \\
\text { Por otra parte, es de recalcar que las } \\
\text { comunidades interpretativas más im- } \\
\text { portantes a la hora de formarse una } \\
\text { opinión con respecto a la violencia } \\
\text { son la familia, los amigos o los gru- } \\
\text { pos de pares en el ámbito laboral. } \\
\text { Abstract } \\
\text { The violence is one of the prob- } \\
\text { lems most worrying for the Salvador- } \\
\text { ians. Daily, the media, in special the } \\
\text { newspapers, relates this problem, }\end{array}$ & $\begin{array}{l}\text { weaves a relation of sense whit the } \\
\text { social perception of violence. In two } \\
\text { years, this investigator studied the phe- } \\
\text { nomenon through a social historical } \\
\text { analysis about the circumstances that } \\
\text { permit this problem get a whirlpool, an } \\
\text { analysis of discourse of ten important } \\
\text { news between } 2003 \text { and } 2007 \text {. The in- } \\
\text { vestigation concludes with an analysis } \\
\text { of reception of three focus groups. } \\
\text { The members of these focus } \\
\text { groups are more critical than we sup- } \\
\text { posed. Their "political capital" gives } \\
\text { more criteria for make their public } \\
\text { opinion about the problems of the real- } \\
\text { ity. I want to emphasize that the com- } \\
\text { munities of interpretation are more } \\
\text { important in the construction of } \\
\text { public opinion than the news- } \\
\text { papers, in special, family, } \\
\text { friends and coworkers. }\end{array}$ \\
\hline
\end{tabular}




\section{Introducción}

- n El Salvador, ocurren por lo - menos diez asesinatos diarios. Las cifras se disparan en muchas ocasiones y llegan a contabilizarse más de veinte. Somos un país pequeño, de 5,7 millones de habitantes, por lo que esta cifra se vuelve alarmante.

Las estadísticas suben y bajan según la institución pública que las maneje, por lo que no es mi afán colocarle un número frío al dolor y al desgarramiento que la violencia, específicamente el aumento en el número de asesinatos, causa en nuestra sociedad.

La violencia es el problema que le atañe a esta investigación. Así de claro. Pero este tema, tan propio de la sociología y la psicología social, también puede abordarse clescle la Comunicación.

El problema que originó esta larga investigación de dos años y medio fue la forma, a mi parecer inadecuada por explicita y carente en muchas ocasiones de profundización y seguimiento, en que los medios de comunicación relatan y retratan la violencia que afecta al país.

La Comunicación tiene mucho que aportar a la reflexión de los problemas de la realidad salvadoreña. Siendo una disciplina recién introducida al país (apenas en los años noventa se abrió la primera carrera de Comunicaciones en la
Universidad Centroamericana "José Simeón Cañas"), existe un amplio número de temas urgentes a los que prestar atención.

Ha llegado el momento en que el estatus científico de la Comunicación ya no debe ponerse en discusión, tal como apunta la comunicóloga argentina Delia Crovi Druetta: "Debido a la resistencia que ha despertado el status científico de la comunicación, basada en la imprecisión de su objeto de estudio, conviene aclarar que, desde mi perspectiva, tal objeto de estudio es claro y lo identifico con el proceso comunicativo que puede ser estudiado de manera global o en alguna parte puntual de sus componentes. Puede asimismo ser analizado desde sus repercusiones sociales, su economía política, sus procesos productivos y estéticos, sus usos y prácticas sociales, las apropiaciones mediáticas, entre otras perspectivas" (Crovi, 2006:25).

De este proceso, a esta investigación le atañe la apropiación que los agentes hacen de los mensajes de la prensa escrita sobre la violencia, es decir: ¿Cuál es la relación de sentido entre la producción de la nota roja en la prensa escrita salvadoreña y la percepción social de la violencia? Pregunta eje.

Sentido entendido como el significado de los discursos, según señala Paul Ricoeur: "Si todo discurso 
se actualiza como acontecimiento, todo discurso es comprendido como sentido" (Ricoeur, 2003:26). Los discursos de los medios cobran sentido a partir de las reinterpretaciones y apropiaciones de los agentes.

Por otro lado, cuando hacemos referencia a la nota roja, aunque en El Salvador se le denomina nota policial o de sucesos, tomamos en cuenta la afirmación del periodista mexicano J.M. Servín que dice que esta es "un producto cultural de arraigue popular (...) un género muchas veces considerado proscrito $y$ bastardo pero un referente ineludible, por lo que evadirlo sería negar su importancia como testimonio" (Servín, 2007).

Cabe subrayar que mi hipótesis inicial ha cambiado radicalmente al término de esta investigación, si bien intentaba describir un fenómeno de insensibilización frente a la violencia, el trabajo de campo mostró que los salvadoreños no han perdido completamente la capacidad de conmoverse ante los asesinatos y son más críticos frente a los medios de comunicación de lo que suponía, sobre todo debido al 'capital político', propio de este pequeño país polarizado, y que consiste en los conocimientos u opiniones formados a partir de la ideología de izquierda o derecha.

Si bien, hemos aprendido a vivir natural y cotidianamente con la violencia.

Por lo que mi hipótesis es que la percepción de este problema es construida socialmente mediante un proceso complejo en el que prima la esfera de la vida cotidiana y las comunidades de apropiación inmediatas a las que pertenecen los agentes, tales como la familia, los amigos y los compañeros de trabajo, más allá de una posible 'influencia' directa de los medios.

\section{Teoría y método}

- I presente trabajo tomó como base metodológica a la "hermenéutica profunda" de John B. Thompson.

Thompson, en su libro "Ideología y cultura moderna. Teoría y crítica social en la era de la comunicación de masas" propugna las tres fases de esta hermenéutica profunda: análisis socio histórico, el análisis formal o discursivo y la interpretación de los anteriores.

En el desarrollo metodológico del enfoque tripartito propone tres niveles de análisis: el de la producción, transmisión o difusión; el de la construcción de los mensajes y el de la recepción y apropiación (Thompson, 2002:445). 
En mi caso, si bien abordo los tres niveles pongo principal interés en el de la recepción, es decir, cómo los agentes resignifican e incorporan a su vida cotidiana los mensajes de los medios de comunicación. Para ello se llevaron a cabo tres grupos de discusión de seis personas cada uno (tres mujeres y tres hombres), utilizando como base para su elección, aparte de la edad y el género, las categorías de capital cultural y económico que según el sociólogo francés Pierre Bourdieu son más abarcadores que una estratificación por clases sociales.

"Las diferencias primarias, aquellas que distinguen las grandes clases de condiciones de existencia, encuentran su principio en el volumen global del capital como conjunto de recursos y poderes efectivamente utilizables, capital económico, capital cultural, y también capital social: las diferentes clases (y fracciones de clase) se distribuyen así desde las que están mejor provistas simultáneamente de capital económico y de capital cultural hasta las que están más desprovistas en estos dos aspectos", señala el autor (Bourdieu, 2005:13).

Por su lado, el aporte de Thompson consiste en enfatizar el carácter simbólico de los fenómenos culturales, y asevera que éstos se insertan en contextos sociales estructurales.

La violencia es simbólica, ha permeado la vida cotidiana y los medios de comunicación, está insertada en un contexto social e histórico complejo el cual no podemos ignorar.
Por lo que también puse énfasis en el marco teórico en abordar el tema de la cultura, el sentido, la construcción social de la realidad, las comunidades interpretativas y de apropiación pero sobre todo la teoría de la estructuración, de Anthony Giddens, uno de mis pilares teóricos fundamentales.

Recalcando, sin duda, que la violencia y su percepción está directamente relacionada con la cultura.

"Creyendo con Max Weber que el hombre es un animal inserto en tramas de significación que el mismo ha tejido, considero que la cultura es una urdimbre y que el análisis de la cultura ha de ser por lo tanto, no una ciencia experimental en busca de leyes, sino una ciencia interpretativa en busca de significaciones. Lo que busco es la explicación, interpretando expresiones sociales que son enigmáticas en su superficie", comenta Clifford Geertz (Geertz, 2005:20).

Hay que profundizar en la cultura y su estudio para comprender el problema de la violencia y su percepción social a través de los medios.

También nos es necesario aclarar qué es una comunidad interpretativa. Según el investigador mexicano Guillermo Orozco, se entiende básicamente "como un conjunto de sujetos sociales unidos por un ámbito de significación del cual emerge una significación especial para su actuación social, en la comunidad de interpretación los agentes sociales se 
convierten en lo que son por medio de su participación en la comunidad" (Orozco, 1991:49).

Las comunidades interpretativas permiten a los agentes construir su opinión con respecto a los problemas de la realidad social y su tratamiento en los medios.

Del análisis de Giddens rescato las condiciones inadvertidas de la acción social, la importancia del registro reflexivo de ésta, su racionalización por parte de los agentes y las consecuencias no buscadas de la acción. Estos conceptos nos ayudan a entender por qué la violencia se constituye en una vorágine en El Salvador, y por qué ocurre su reproducción sistémica. Como condiciones inadvertidas podemos mencionar la pobreza y la exclusión social y como consecuencias no buscadas de la acción, el uso de las armas de fuego.

Según informes del Programa Nacional de las Naciones Unidas para el Desarrollo (PNUD) existen por lo menos 450.000 armas en manos de civiles.

Giddens agrega: "La reificación de las relaciones sociales, o la 'naturalización' discursiva de las circunstancias y productos históricamente contingentes de la acción humana, es una de las dimensiones principales de la ideología en la vida social" (Giddens, 1996:62).

La naturalización discursiva de la violencia es uno de los problemas prácticos que me hizo iniciar esta investigación. El que los agentes se acostumbren a estar rodeados de violencia, y aprendan a vivir en un país con tan alto número de asesinatos.

Esto se manifiesta en situaciones tan dramáticas, como el hecho de que cada salvadoreño si no ha sido víctima de la violencia, conozca por lo menos a un familiar o amigo que ha sido asesinado o por lo menos asaltado.

Y contrasta con el hecho de que el porcentaje de victimización, según recientes encuestas del Instituto Universitario de Opinión Pública (IUDOP), alcanza un 13 por ciento. Es decir, que de cada cien consultados, solo trece han sufrido en carne propia los embates de la delincuencia (Cruz y Santacruz, 2005).

Las autoridades del Consejo Nacional de Seguridad Pública (CNSP), consultadas para la presente investigación, han insistido en que la violencia es un problema focalizado en ciertos municipios y no una problemática generalizada como lo percibe la población.

Siguiendo con el desarrollo teórico, es muy importante recalcar que el concepto de agente, incluido en la teoría de la estructuración, es fundamental para esta investigación. Al hacer referencia a los ciudadanos salvadoreños, los llamamos agentes.

"Esto presupone que ser un agente es ser capaz de desplegar (repetidamente, en el fluir de la vida diaria) un espectro de poderes causales, incluido el poder de influir sobre el desplegado por otros. Una 
acción nace de la aptitud del individuo para 'producir una diferencia' en un estado de cosas o curso de sucesos preexistentes. Un agente deja de ser tal si pierde la aptitud de 'producir una diferencia', o sea, de ejercer una clase de poder" (Giddens, 1996:51).

En este punto y explicando la metodología utilizada, al haberme embarcado en una investigación de tres niveles, el trabajo se triplicó pues la tesis está estructurada de la siguiente manera: un análisis sociohistórico de las circunstancias que originaron la violencia actual, un capítulo teórico doncle sustento los conceptos utilizados que conllevaron al diseño de un instrumento para el análisis de recepción y un capítulo que incluyó el análisis de discurso de diez notas emblemáticas de violencia durante los últimos años (2003-2007, en El Diario de Hoy y La Prensa Gráfica) y la consulta con los productores de la nota roja para conocer el proceso de producción de los mensajes (editores y fotoperiodistas).

Mediante el análisis sociohistórico se recoge -a través de múltiples textos históricos- los antecedentes de la vorágine de violencia en El Salvador desde el etnocidio ordenado por el dictador Maximiliano Hernández Martínez en 1932 que habría dejado al menos 30.000 víctimas.

"La matanza fue espantosa, las fuerzas represivas del gobierno y las guardias cívicas ametrallaban a los campesinos indiscriminadamente en las estaciones de ferrocarril, en las calles de los pueblos del interior, en los caminos vecinales, en las carreteras, en las fincas y haciendas" (Dalton, 2006:106), describe el poeta Roque Dalton en su monografía sobre El Salvador.

"Una paz de cementerio" (Dalton, 2006:109), falsa y débil, es lo que el dictador Hernández Martínez habría conseguido con el etnocidio de 1932, apuntó. Una paz que décadas después se convertiría en su total antagonista.

Por lo que también, en el cuerpo de la investigación se aborda la violencia ocasionada por la guerra civil salvadoreña (1980-1992), y las circunstancias históricas que nos llevaron hasta la inseguridad ciudadana actual.

En segunda instancia, las notas emblemáticas elegidas fueron -por su importancia para la opinión pública-: el asesinato de la niña Katia Miranda, el aparecimiento de mujeres descabezadas, el aumento del número de asesinatos y de los operativos contra las pandillas, los 31 reos que murieron en el penal de Mariona, el asesinato del niño Maycol José Ticas y el homicidio de dos policías a manos de un sujeto armado frente a la universidad nacional, así como notas de seguimiento de estos sonados casos.

Para el análisis del discurso me basé en el autor Teun A. van Dijk, quien posee un enfoque práctico, pedagógico y claro a la hora de su aplicación. 
A continuación presentamos al lector una de las diez notas analiza-

das, para que tenga una idea clara del análisis realizado:

\begin{tabular}{|l|}
\hline Título de la nota \\
Arrecia lucha contra las \\
pandillas a nivel nacional. \\
\\
\\
\hline Comentario sobre la cohe- \\
rencia de la nota (depen- \\
diendo de si se pudo dedu- \\
cir tema o asunto) \\
En general, sí hay una co- \\
herencia entre el título de la \\
nota y el tema de ésta, aun- \\
que no queda claro del todo \\
por qué arrecia la lucha con- \\
tra las pandillas pues no hay \\
suficientes elementos de con- \\
texto para comparar las ante- \\
riores acciones y las nuevas.
\end{tabular}

Tema o asunto

El Plan Súper Mano

Dura para combatir a

las pandillas.
Macroestructura o macroproposición (luego de aplicar las macrorreglas)

El gobierno y la policía impulsaron varias medidas como parte del plan Súper Mano Dura para combatir a las pandillas durante el año 2004.

\section{Las proposiciones más importantes luego de aplicar las} macrorreglas.

Al tomar su mandato en julio el Presidente Antonio Saca puso en marcha el plan Súper Mano Dura con el fin de desbaratar las estructuras de las pandillas en El Salvador.

El gobierno y la policía impulsaron varias medidas como parte del plan Súper Mano Dura para combatir a las pandillas (esta es la macroestructura) con la participación de 14.000 policías, reformas al código penal, mesas de trabajo con distintos sectores, la creación de los Grupos de Tarea Antipandillas (GTA) y reacomodos en cuatro cárceles del país. Cuatro mil miembros de pandillas han sido arrestados por las autoridades en el país durante las operaciones.

Propiedades del contexto $o / y$ representaciones o factores socioculturales

Como en las anteriores dos notas, el periodista no da suficientes datos del contexto de esta información, no hay un "background" para que el lector que por primera vez se acerca a este tema tenga claro por qué ha arreciado la lucha contra las pandillas ¿En comparación con qué medidas? Tampoco se comenta algo muy importante y es que tanto el Plan Mano Dura como el Plan Súper Mano Dura fueron muy cuestionados por organizaciones internacionales y de la sociedad civil por violar los derechos universales de libre asociación o imagen, puesto que se legalizó la detención de personas por el solo hecho de usar tatuajes, además la ley contemplaba que más de dos jôvenes reunidos en una esquina eran "asociaciones ilícitas". Algunos organismos humanitarios en el país llegaron a afirmar que se perseguía y penalizaba ser joven en El Salvador.

\section{Persuasión}

En esta nola subyace el diálogo persuasivo de querer convencer al lector de que el gobierno y la policía estaban luchando arduamente contra la inseguridad ciudadana, en esta época, y aún ahora, las encuestas reflejaban que uno de los problemas que más preocupa a la población salvadoreñas es la inseguridad. Este diálogo persuasivo se puede ver en el encabezado que señala "de día y de noche las fuerzas clel orclen público no permitieron espacio a las maras en el territorio". Hay una intención de presentar a la autoridad como héroe, se cae además en una falacia pues al decir que ni de día ni de noche se permitió el espacio a las maras en el territorio ( 2 Cuál territorio? esta afirmación es muy vaga) es como si ya no existieran. Además, la nota justifica las reformas legales (y no incluye la reseña de la crítica que causaron éstas hasta en el mismo Organo Judicial). Esta nota está cargada de personalización, en contra de lo que ya mencionamos que debe caracterizar al género seco.

Observaciones: Hay coherencia, se cumple la proyección semántica, no así, la labor del periodista en lo que refiere a la despersonalización del discurso en el género seco o informativo. 
En algún momento, claro, pensé en acortar recursos y esfuerzos, y quedarme solo en alguno de los tres niveles de análisis, pero el tema urgía ser tratado con profundidad y visión holística. Así, cumplí con lo propuesto metodológicamente por Thompson y su "hermenéutica profunda".

Con respecto al estado del arte de este tema hay dos estudios que considero básicos y pioneros en el área y forman parte de mis antecedentes.

El primero, el realizado por José Miguel Cruz y María Santacruz Giralt, La victimización y la percepción de seguridad en El Salvador en 2004 (Cruz y Santa Cruz, 2005), que es parte de una iniciativa gubernamental y de distintas organizaciones internacionales y de la sociedad civil para medir la relación entre los índices de victimización y la percepción de la violencia.

Por primera vez, en El Salvador se estudió directamente el papel de los medios de comunicación en la construcción de esta percepción. El estudio es cuantitativo, basado en una encuesta a 2.300 personas, y bastante esclarecedor.

En segundo lugar, el libro Violencia y Medios III, Propuesta Iberoamericana de Periodismo Policial
(Klahr y Portillo, 2007); forma parte de una serie de tres volúmenes y constituye una iniciativa de periodistas preocupados por el tratamiento de la violencia; se plantea el "deber ser" del hacer periodístico y critica la forma, la imprecisión y la falta de ética con que, en muchas ocasiones, se aborda el tema.

Amparo Marroquín, investigadora salvadoreña y comunicóloga, participa en este proyecto con un ensayo titulado Pandillas y prensa en El Salvador. De los medios como oráculos y de la profecía que se cumplió con creces donde realiza un análisis de discurso sobre el tema de las "maras" y la construcción de estereotipos sobre estos grupos.

La bibliografía sobre violencia, es amplísima, y en esta investigación se han recogido algunos autores que han abordado la problemática clesde el psicoanálisis y la psicología social.

Pero como han podido leer, el presente trabajo es muy particular en el tiempo (2003-2007), el lugar (El Salvador) y el sentido que se establece entre medios de comunicación y percepción social de la violencia. Esta tesis parte desde la Comunicación para dar su aporte.

\section{Apuntes finales}

Los principales resultados de este trabajo se derivan del análisis de discurso y del análisis de recepción que coronan el documento 
final. Del primer capítulo, es muy importante rescatar que sin la comprensión de los hechos históricos que antecedieron a la actual vorágine de violencia no podremos comprender el problema ni dibujar una posible solución a esta crisis social.

Enumero a grandes rasgos las principales conclusiones de mi tesis:

-Los medios de comunicación, y en particular la prensa escrita, contribuyen a establecer la agenda de los temas (incluida la violencia) que preocupan a los salvadoreños, pero no determinan las opiniones que al respecto se van formando éstos. Estas se ven más influenciadas por el criterio personal formado en comunidades interpretativas tales como la familia, los amigos y el trabajo.

-Sin embargo, su papel no puede subestimarse porque los medios sí han contribuido a elevar la percepción de inseguridad y la sensación de que la violencia es un problema generalizado. El nivel de victimización no necesariamente corresponde con la percepción de inseguridad y esto se le atribuye en gran medida a los medios de comunicación que estarían manteniendo a la población en alerta y preocupación constante.

-Con respecto al discurso, los productores de nota roja en la prensa escrita si bien mantienen un discurso periodístico coherente, no están tratando el tema con estricto profesionalismo y responsabilidad social. Muchas veces no contextua- lizan suficiente los hechos u opinan en notas informativas de género seco. Es decir, no respetan los manuales básicos de periodismo.

- La forma en que la prensa escrita salvadoreña está abordando la violencia se queda muchas veces en el relato, sin preocuparse lo suficiente en investigar las causas del problema.

-No obstante, ha iniciado un proceso de racionalización de la labor periodística, los medios de comunicación y sus trabajadores de la palabra han empezado a reflexionar sobre la importancia de la forma en que abordan el tema. Los productores de la nota roja han hecho consciente la importancia de su papel a la hora de abordar el problema y en un medio escrito en particular (La Prensa Gráfica) se echó a andar un proyecto editorial para narrar de una forma más ética y profesional la violencia.

- Los receptores construyen su percepción de la violencia de maneras más complejas que las que suponía al inicio de esta investigación, son mucho más críticos ante los medios, si bien esto depende de su capital cultural y económico, aún en personas que no estudiaron ni siquiera la primaria completa se descubre una profunda postura crítica con respecto a la forma en que los medios de comunicación abordan la violencia. Recalco la importancia de que los agentes cuenten con una clara ideología política o capital político, como un aspecto más que participa en la construcción de sus opiniones. 
- Los medios de comunicación deben de intentar responder a las expectativas de profesionalismo, responsabilidad y ecuanimidad que solicitan sus lectores a la hora de abordar este tema.

-Durante toda la investigación llamamos a los ciudadanos, agentes. $Y$ en el fondo de este trabajo académico persiste la idea de que éstos

\section{Bibliografía}

Bourdieu, Pierre 1999 (1961) La distinción. Criterio y bases sociales del gusto (Madrid: Taurus).

Crovi, Delia 2006 Educar en la era de las redes (México DF: UNAM).

Cruz, José Miguel y Santacruz Giralt, María 2005 La victimización y la percepción de seguridad en El Salvador en 2004 (San Salvador: UCA Editores).

Dalton, Roque 2006 (1989) El Salvador. Monografía (San Salvador: UCA Editores).

Dalton, Roque 1997 Las historias prohibidas del pulgarcito (México: Siglo XXI Editores).

Geertz, Clifford 2005 (1973) La Interpretación de las culturas (Barcelona: Gedisa).

Giddens, Anthony 1995 (1984) La constitución de la sociedad. Bases para la teoría de la estructuración (Buenos Aires, Amorrortu editores). pueden cambiar las circunstancias que los rodean, en este caso, pueden detener la violencia en El Salvador. Esto no puede blandirse como una iniciativa de individuos, sino que dependerá de la articulación de esfuerzos del gobierno, la sociedad civil, los organismos internacionales, las instituciones públicas y, por supuesto, los medios de comunicación.

Lara Klahr, Marco y Portillo Vargas 2007, Ernesto Violencia y Medios 3. Propuesta Iberoamericana de Periodismo Policial. (México DF: Insyde Ideas)

Orozco Gómez, Guillermo 2001 Televisión, audiencias y educación (Colombia: Editorial Norma).

Orozco, Guillermo 1995 Recepción Televisiva. Tres aproximaciones y una razón para su estudio (México: Universidad Iberoamericana).

Ricoeur, Paul 2003 Teoría de la interpretación. Discurso y excedente de sentido (México: Siglo XXI).

Servín, J.M. 2007 La nota roja, desentrañar identidades proscritas, Ponencia presentada en el ciclo de conferencias 'El cuerpo y lo siniestro en la cultura' organizado por el Instituto Nacional de Antropología e Historia a través del Instituto Nacional de la Di- 
rección de Estudios Históricos, en el centro de Tlalpan, México D.F. el 24 de agosto.

Thompson, John B 2002 (1990) Ideología y cultura moderna.
Teoría crítica social en la era de la comunicación de masas (México: Universidad Autónoma Metropolitana) 